\title{
Efficacy of Psychological Interventions Towards the Reduction of High-Risk Sexual Behaviors Among People Living with HIV: A Systematic Review and Meta-analysis, 2010-2020
}

\author{
Hanxi Zhang ${ }^{1} \cdot$ Qing $\mathrm{Yu}^{2} \cdot{\text { Zheng } \mathrm{Li}^{1} \cdot \text { Xiangfei Xiu }^{1} \cdot \text { Fan Lv }^{1} \cdot \text { Mengjie Han }}^{1} \cdot$ Lu Wang $^{1}$ \\ Accepted: 30 January 2021 / Published online: 8 February 2021 \\ (๑) The Author(s), under exclusive licence to Springer Science+Business Media, LLC part of Springer Nature 2021
}

\begin{abstract}
People living with HIV/AIDS (PLWH) may be vulnerable to mental illness. As sexual transmission is the leading cause of HIV infection, evidence-based study for the effect of psychological interventions on the change of sexual is needed. To estimate the efficacy of psychological interventions towards reducing unprotected sex and increasing condom use among PLWH. We systematically searched PubMed, Web of Science, EMBASE (OVID), and PsycINFO (OVID) for studies reporting psychological intervention effects on the outcomes of condom use and/or unprotected sex from 2010 to 2020 . This review is registered with PROSPERO, CRD42020193640. Of 949 studies, 17 studies were included in this systematic review. Overall, participants in the intervention group reduced sexual risk or condomless sex relative to control groups. The effect was higher for people having sex with HIV-positive partners comparing with those who had sex with HIV-negative or unknown status partners. Psychological interventions might positively affect the condom use of PLWH and should be prioritized and regularly.
\end{abstract}

Keywords HIV $\cdot$ Behavior $\cdot$ Condom use $\cdot$ Psychological interventions $\cdot$ PLWH $\cdot$ Meta-analysis

\section{Introduction}

HIV has become a chronic illness instead of a disease that rapidly leads to severe sickness or death due to antiretroviral medication in recent years. Sexual transmission, via unprotected heterosexual or homosexual contact, is the leading cause of HIV infection. Thus, sexual risk behaviors increasing becomes an important reason for HIV transmission [1]. Simultaneously, people living with HIV/AIDS (PLWH) are those who suffer mental illness most [2]. Depression or stigmas occur commonly in HIV-positive individuals with limited the quality of life, and leading to poorly clinical outcomes [3]. A study focused on mental health problems among female sex workers has revealed that sexual assault was associated with current PTSD (post-traumatic stress

Lu Wang

wanglu64@163.com

1 National Center for AIDS/STD Control and Prevention, Chinese Center for Disease Control and Prevention, No.155 ChangBai Road, Beijing 102206, China

2 Department of Xiamen Cancer Prevention and Control, The First Affiliated Hospital of Xiamen University, Teaching Hospital of Fujian Medical University, Xiamen, China disorder) symptoms. Female sex workers who had experienced violence were five times more likely to be depressed than those who were not victims of violence [4], which may increase the spread of HIV or sexual transmitted diseases (STDs) [5]. HIV-related stigma might lead HIV-positive people to internalize negative messages about themselves and other HIV-positive individuals [6]. The internalized HIV-related stigma might lead to HIV transmission-related risk behaviors, including suboptimal antiretroviral adherence, condomless sex, avoidance of medical services, and HIV status disclosure [7].

In light of the above, several previous psychosocial interventions were conducted recent years to promote mental health among PLWH. Theoretical basis including cognitivebehavioral therapy (CBT), stress management interventions, information-motivation, and behavioral skills (IBM) have been developed for PLWH with mental disorders. These interventions effectively reduce symptoms of depression, anxiety and improving quality of life among PLWH $[8,9]$. At the same time, previous systematic reviews and metaanalyses about behavioral intervention suggested that risk reduction interventions on people living with HIV/AIDS are efficacious in reducing $43 \%$ of self-reported unprotected sex $[1,10]$. However, no meta-analysis was conducted to 
investigate the effects of psychological interventions on sexual behaviors.

Evidence showed that condom effectiveness against HIV found an average protective effect of $80 \%$ for consistent condom use, unadjusted for lack of correct use [11]. One study in New Zealand have emphasized evidence-based primary prevention by condom use, which was implemented in communities and supported by comprehensive public health action [12]. Condom use remains a staple prevention method though international attention has been recently devoted to biomedical prevention strategies such as pre-exposure prophylaxis (PrEP) $[11,13,14]$.

In conclusion, the systematic review and meta-analysis on psychological interventions was conducted to evaluate the efficacy of HIV risk reduction, especially on condom use, among PLWH.

\section{Methods}

This review complied with preferred reporting items for systematic review and meta-analysis (PRISMA) [15]. A systematic literature search was performed to identify trials that evaluated the impact of psychological interventions on selfreported condom use among PLWH. The literature searching strategy was decided after several rounds of discussion. Two authors performed the searching process independently. Articles with different opinions were discussed with one or more of the other authors to decide.

\section{Literature Search, Study Selection, and Data Extraction}

We searched on PubMed, Web of Science, EMBASE (OVID), and PsycINFO (OVID) from May 4th to 12th, 2020. The search strategy can be found in the Appendix. And We selected studies carried out from January 1, 2010, to December 31, 2019. The articles were screened to determine by the following criteria: (1) The intervention target population was 14 years old or above HIV-positive subjects (including HIV discordant couples). (2) The study design should be intervention studies, including clinical trials, randomized controlled trials (RCT), a quasi-experimental design with a comparison condition, and pilot study. (3) Psychological intervention, which means the intervention primary aimed to change the mental disorders, thoughts, or emotions among PLWH. (4) Intervention outcomes included at least one behavior change technique or behavioral outcomes (condom use, sexual behavior times, number of sexual partners, commercial behavior, etc.) The flow diagram for the identification and selection of articles for inclusion was shown in Fig. 1.
Two trained coders independently coded each eligible intervention for the following study characteristics (e.g., study date, author, location, sample size, study design), intervention characteristics (e.g., components, delivery method, follow-up), outcomes, and risk of bias. If sexual behavior data for different types of partners were reported, the review summarized the results of sex with all partners or at-risk partners (i.e., HIV-negative or status-unknown partners). Besides, for studies that reported multiple measurements or follow-up assessments, we summarized all the results for interventions by different assessments in each eligible research (i.e., condom use frequencies/proportions, condomless sex frequencies/proportions, risk/odds ratio).

\section{Quality Assessment}

Study quality was assessed using adapted Cochrane riskof-bias variables [16]. Each intervention was evaluated for Sequence generation, Allocation concealment, Blinding (participants), Incomplete outcome data, Selective outcome reporting, Availability of treatment manual, and Use of training for therapy providers. Each item was scored as high $(-1)$, or unclear (0) or low (1) risk of bias. Overall, study quality was scored from 0 to 7 , with a higher score indicating a lower risk of bias [17]. 'Low risk of bias', means plausible bias unlikely to seriously alter the results (Score: $\geq 4$ ). 'Unclear risk of bias', means plausible bias that raises some doubt about the results (Score: -4 to 4 ). 'High risk of bias', means plausible bias that seriously weakens confidence in the results (Score: $\leq-4$ ). Disagreements in quality assessment were resolved through discussion.

\section{Data Analysis}

Meta-analysis was conducted among studies using the same measurement of condom use. For studies using various assessment were qualitatively summarized. However, we did not conduct meta-analysis for all included studies due to various intervention design and outcome assessments across sexual behaviors. We used standardized mean differences (SMD) for condom use proportion to calculate the effect size. The SMD was defined as the mean difference between treatment and control groups divided by the pooled standard deviation. Cohen's D was calculated as a measure of effect size, and the evaluation criteria of Cohen's D were as follows: small effects $(\geq 0.2$ and $<0.5)$; Moderate effect $(\geq 0.5$ and $<0.8)$; Big effect $(0.8$ or higher). Heterogeneities were assessed by $I^{2}$ statistics, $I^{2} \leq 50 \%$ was considered as no heterogeneity, and the fixed-effects model was adopted; otherwise, a random-effects model with restricted maximum likelihood variance estimation was used to obtain average condom use effect sizes. And the funnel plot, Egger's test of the intercept, was employed to assess indications of 
Fig. 1 Flow chart showing the search and study selection process for literature

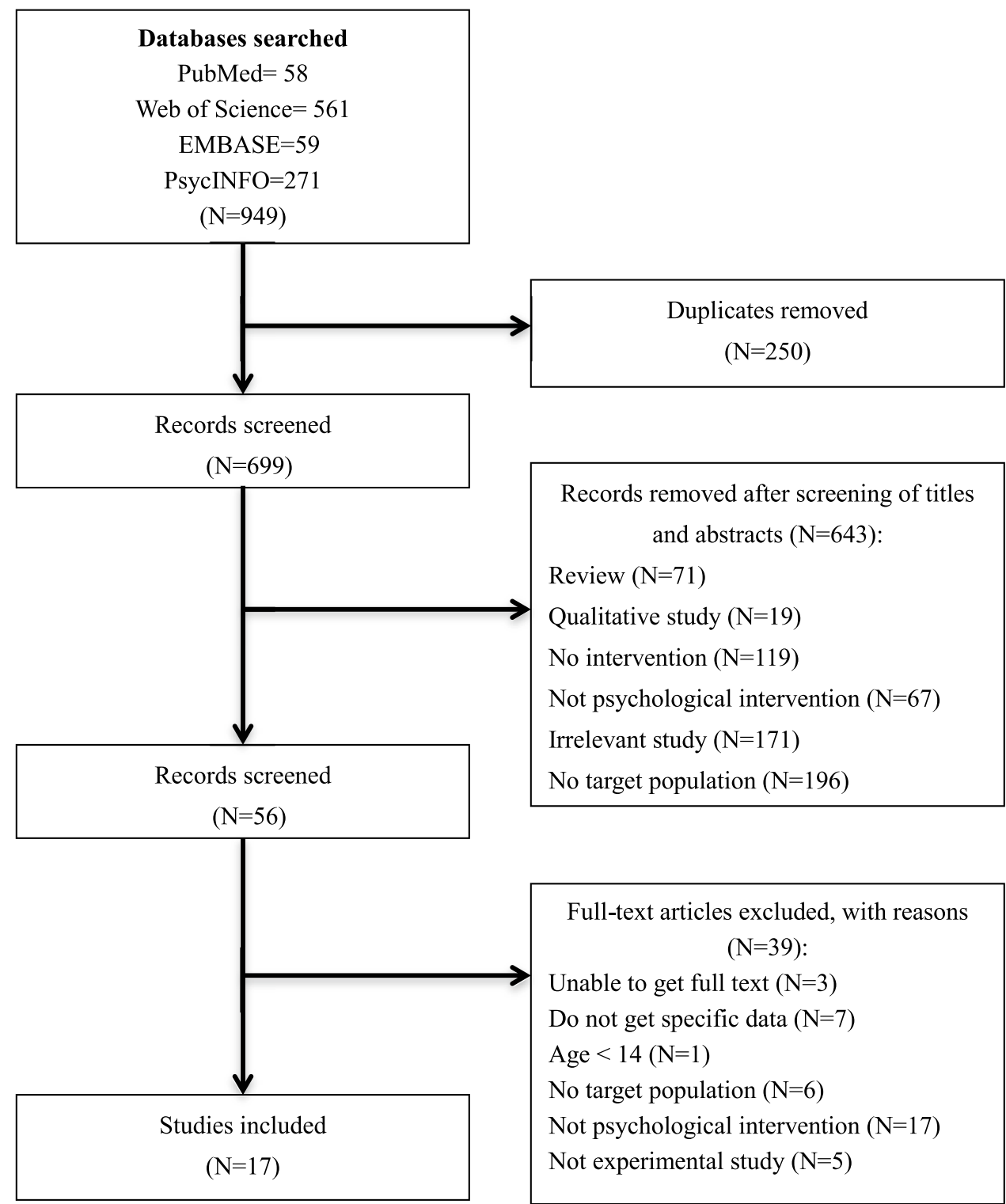

publication bias. All analyses were conducted in R studio 1.1.456 using a "meta" meta-analysis package.

\section{Results}

Database searching produced a total of 949 citations (Fig. 1). After the initial screening of duplicates, 699 citations were dependently reviewed by two authors, of which 643 were excluded for not meeting the inclusion criteria (e.g., review, qualitative studies, irrelevant studies, or studies without psychological intervention). Fifty-six articles were pulled for full-text review, and 39 were excluded for different reasons (unable to access the full text or specific data, or no target population, et al. See details in Fig. 1). Ultimately, 17 studies were included in the review (Table 1). The review registration number was PROSPERO CRD42020193640.

\section{Study Descriptions}

As Table 1 showed, included studies were published between 2010 and 2020. In total, 2885 participants reported in 17 unique publications [18-34] were included in the current review. Interventions focused on HIV-positive men who have sex with men (MSM) $(n=6)$, HIV discordant couples $(n=2)$, HIV-positive women $(n=2)$, or other HIV-positive participants $(n=7)$. The majority of the interventions were conducted in the United States $(n=8)$, while others were conducted in South Africa $(\mathrm{n}=1)$, Nepal $(\mathrm{n}=1)$, Canada $(n=1)$, Malawi $(n=1)$, Europe $(n=1)$, and four unclear study fields. 
3358

AIDS and Behavior (2021) 25:3355-3376

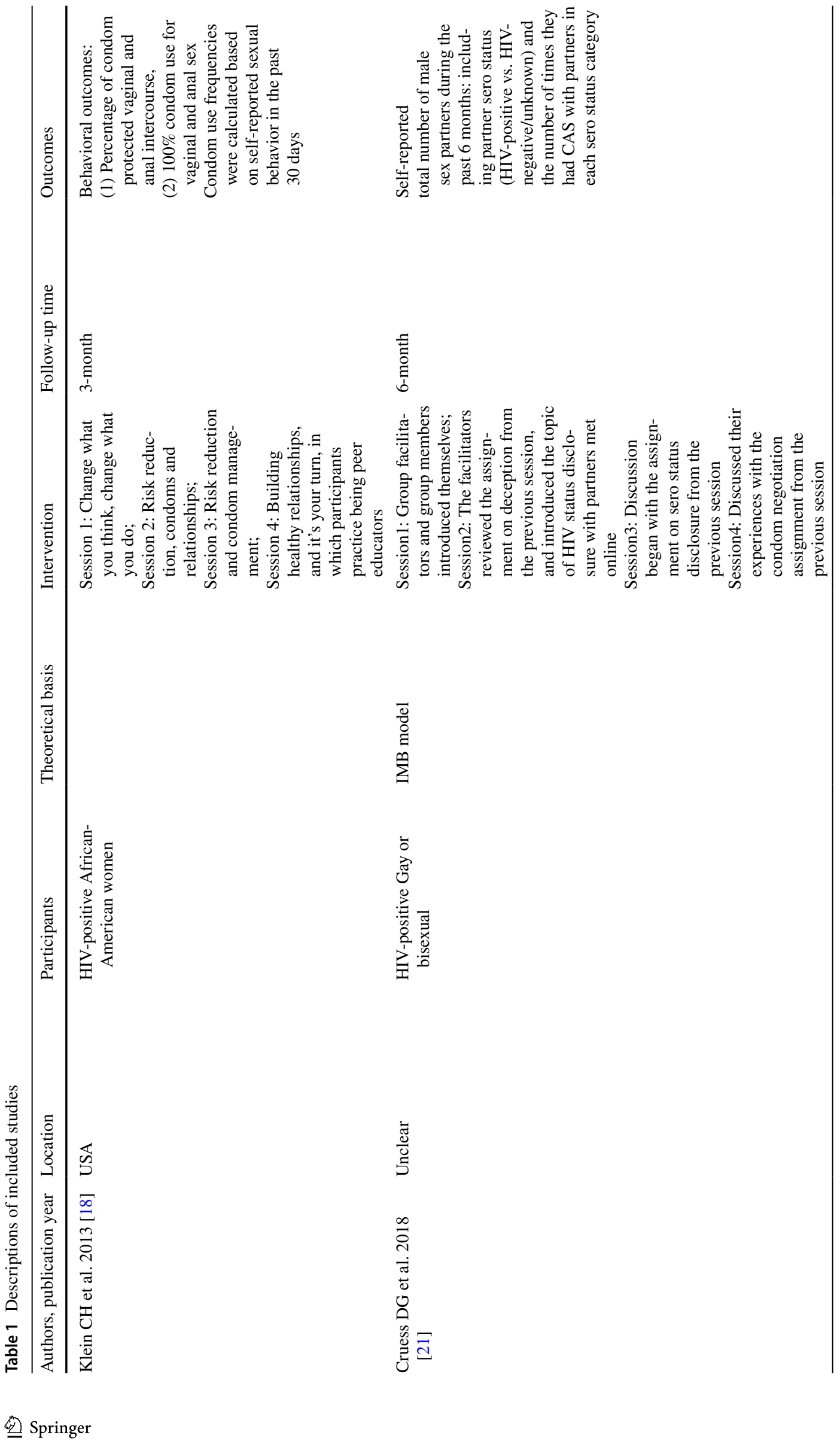




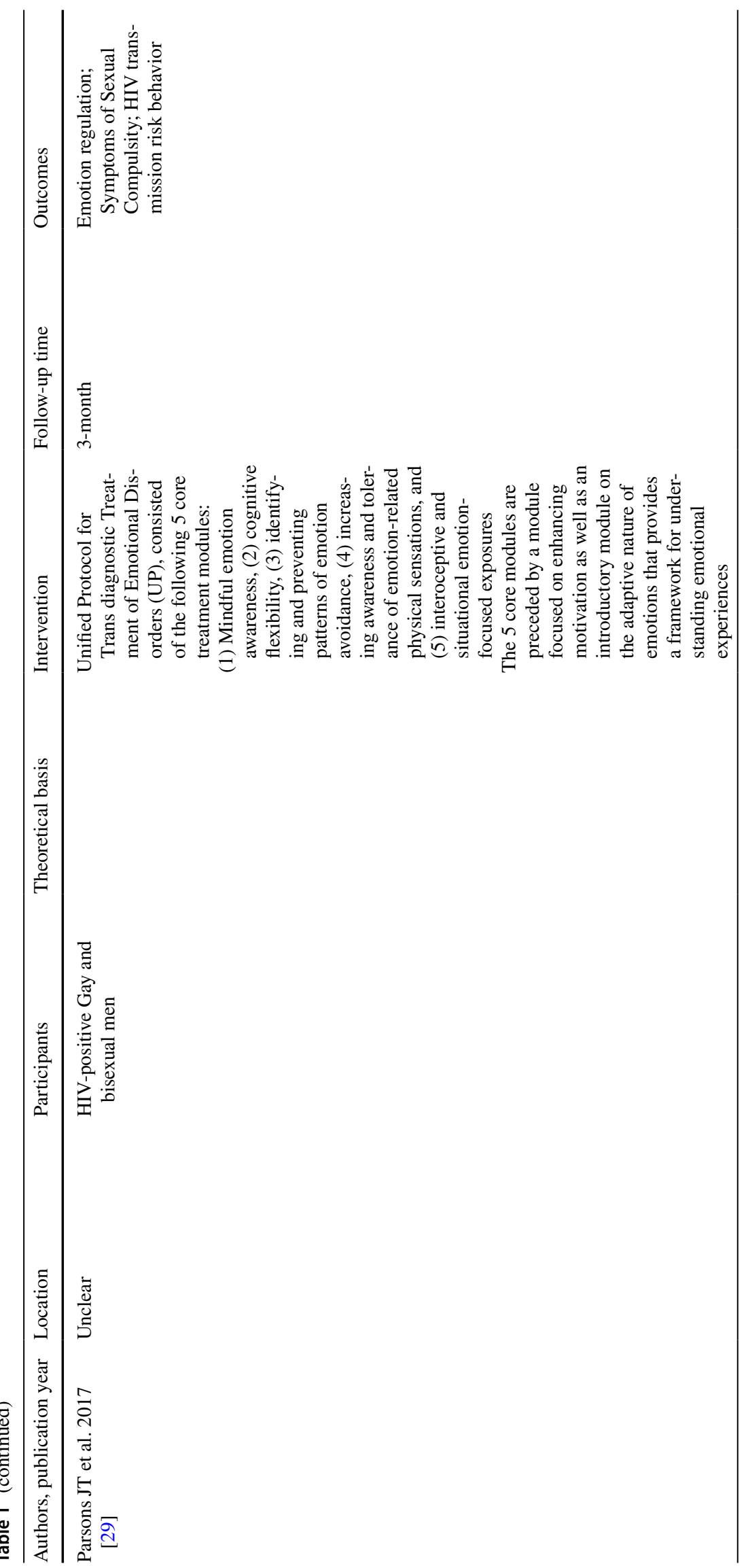




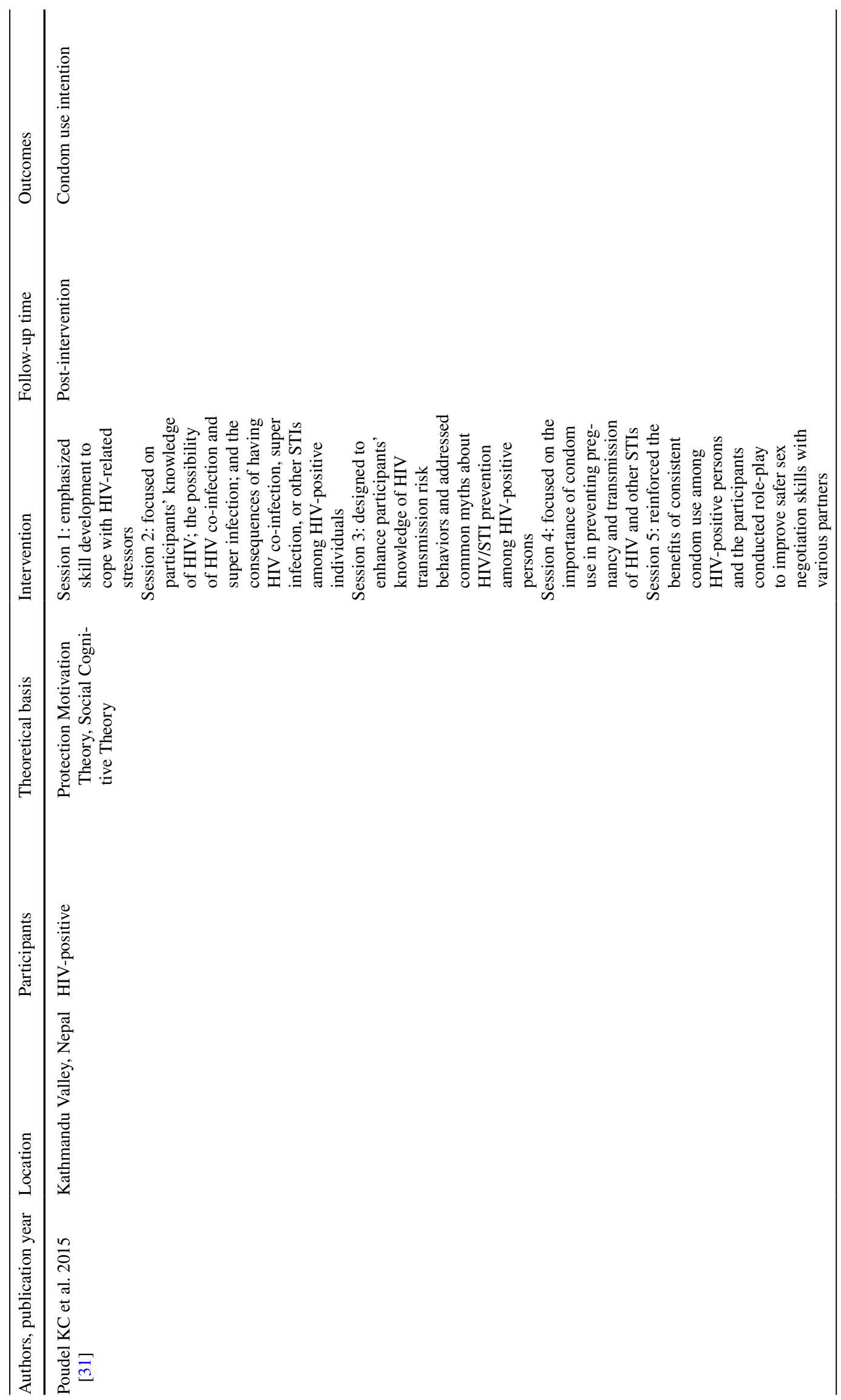




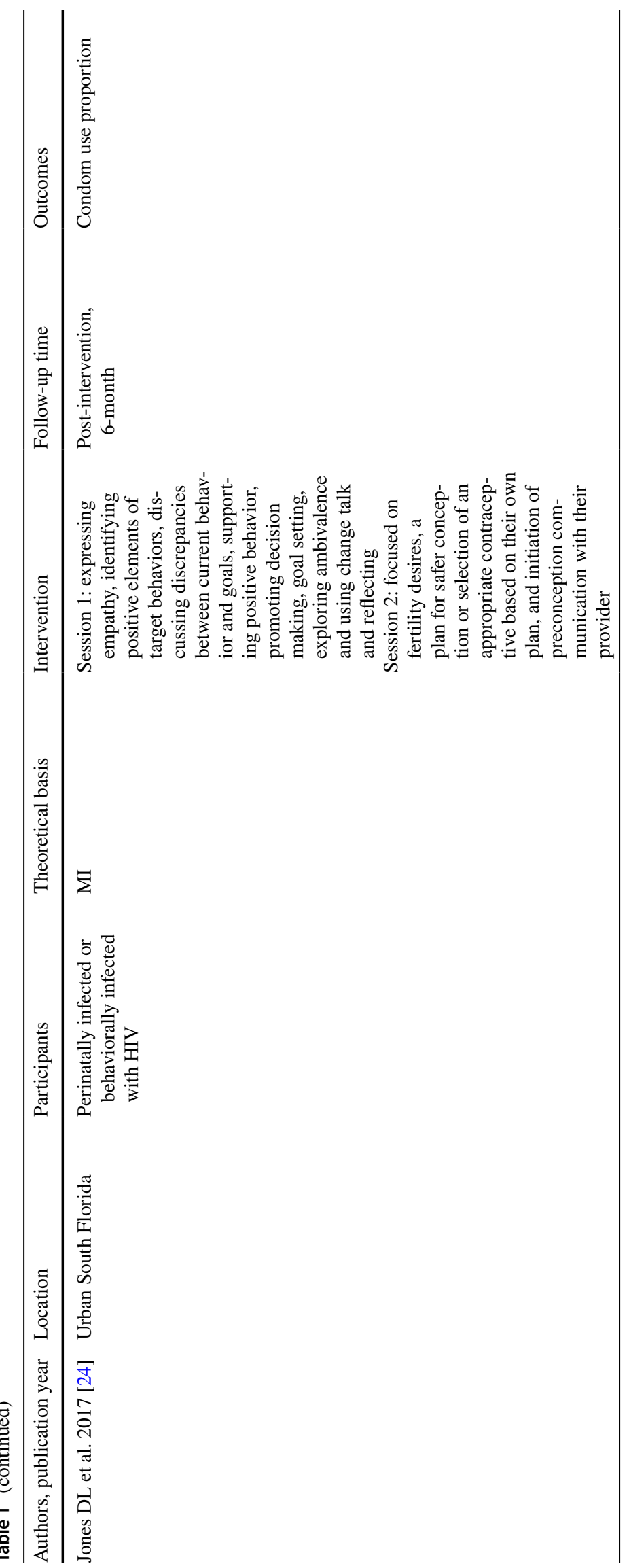




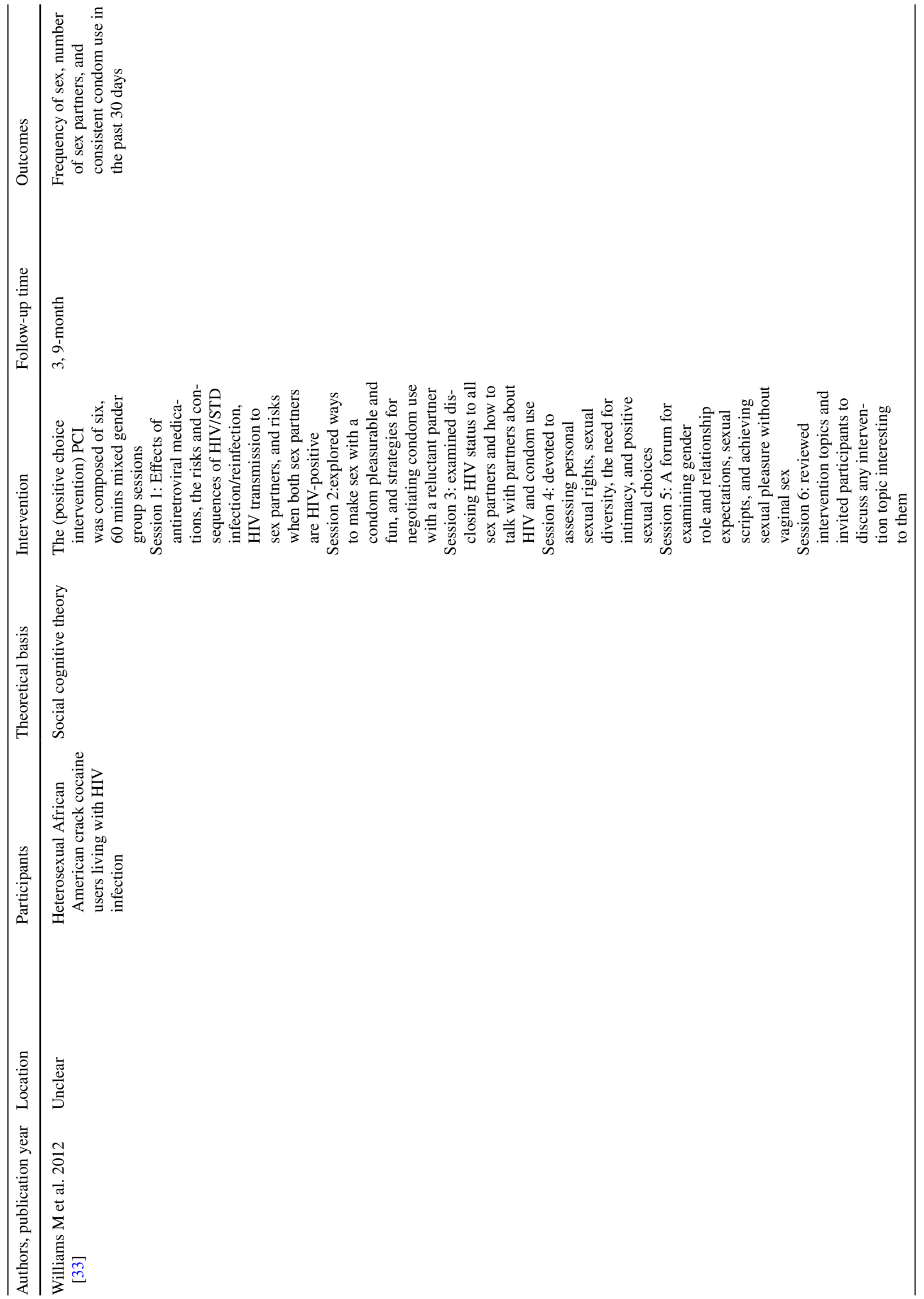




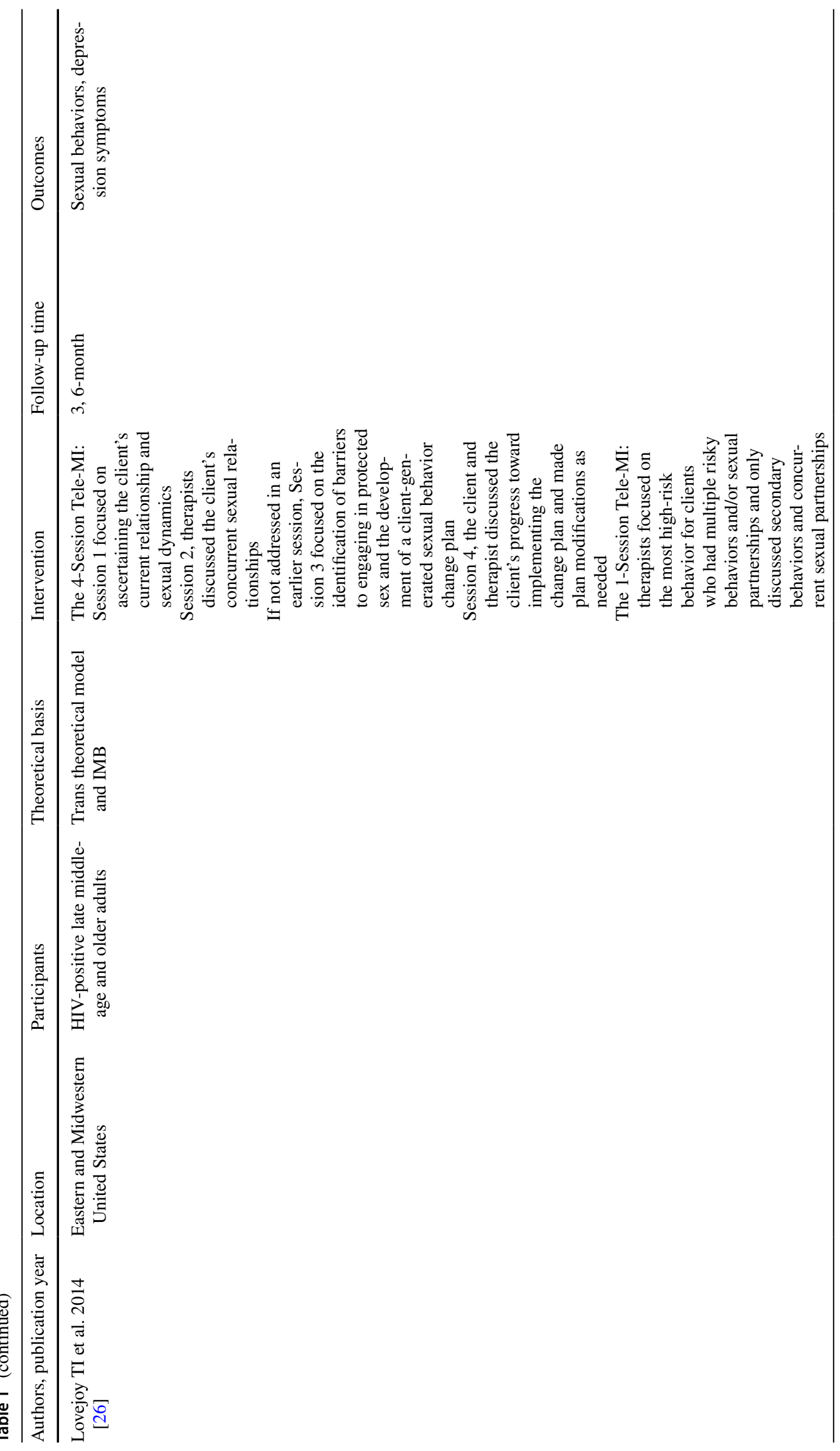




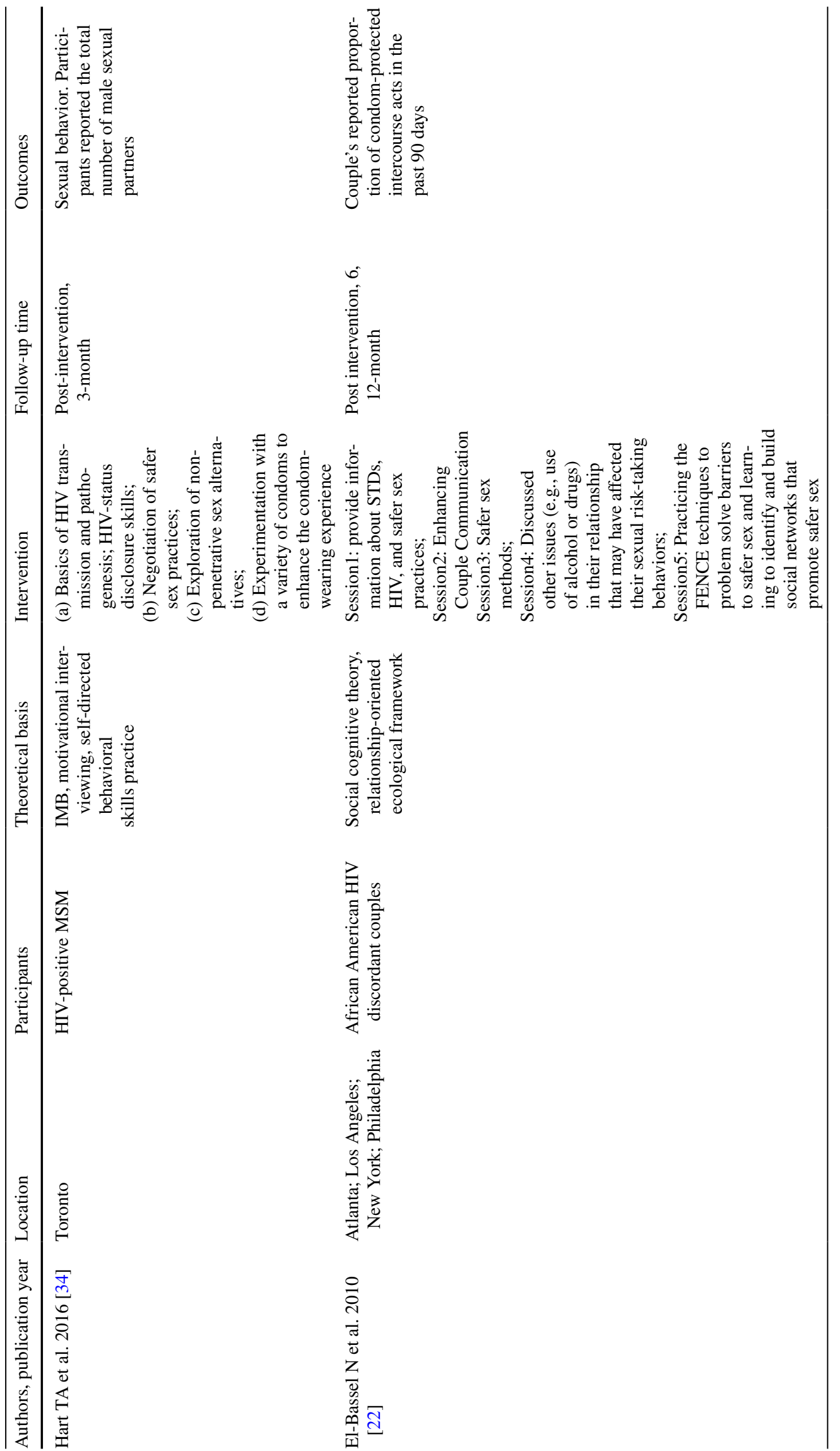




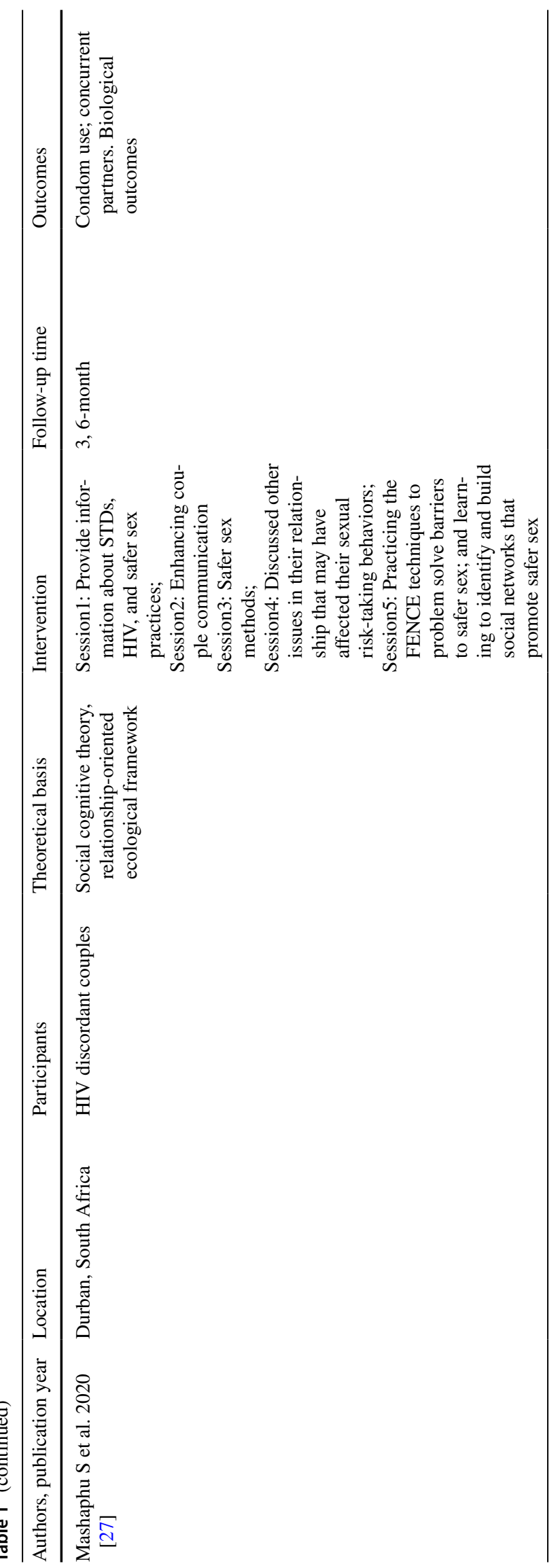




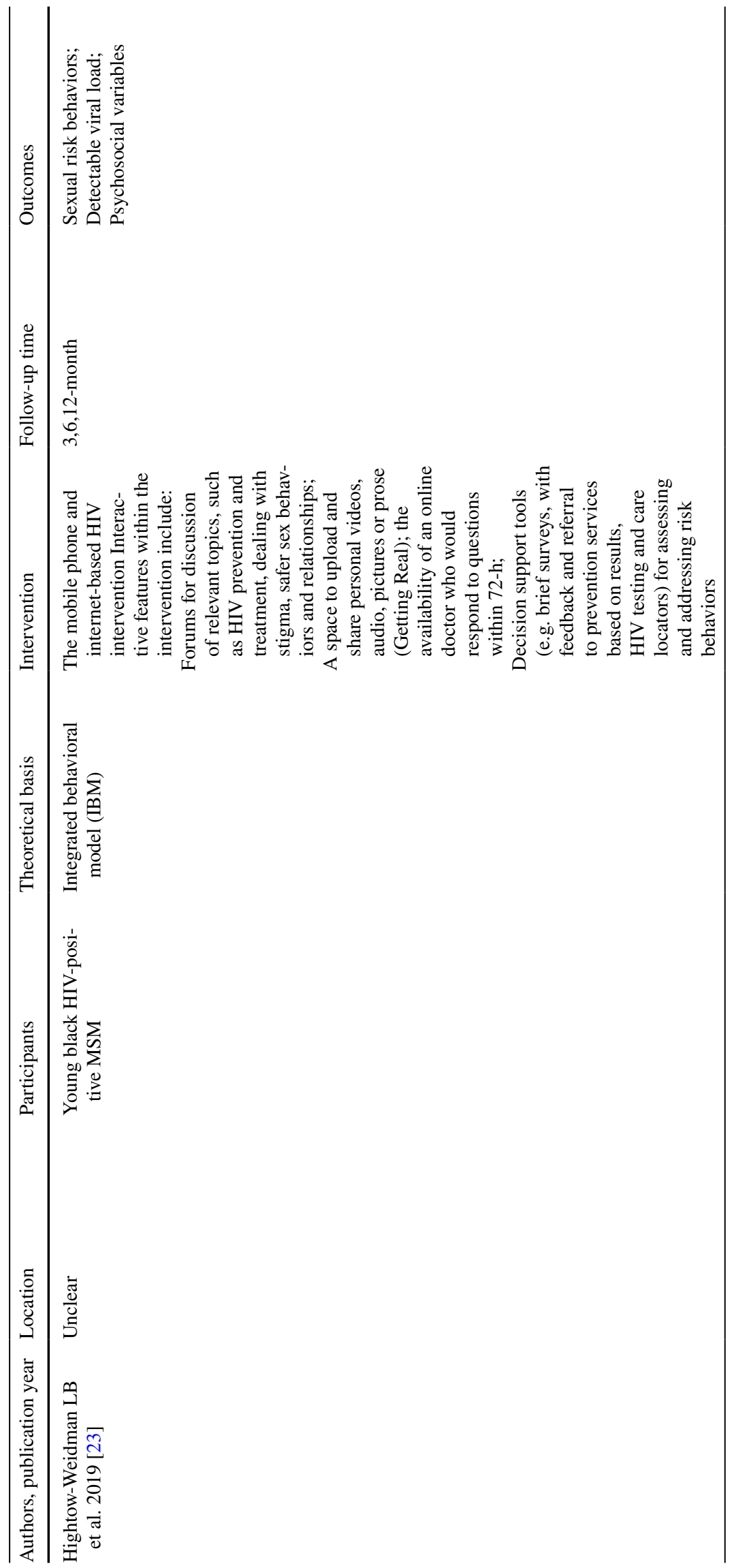




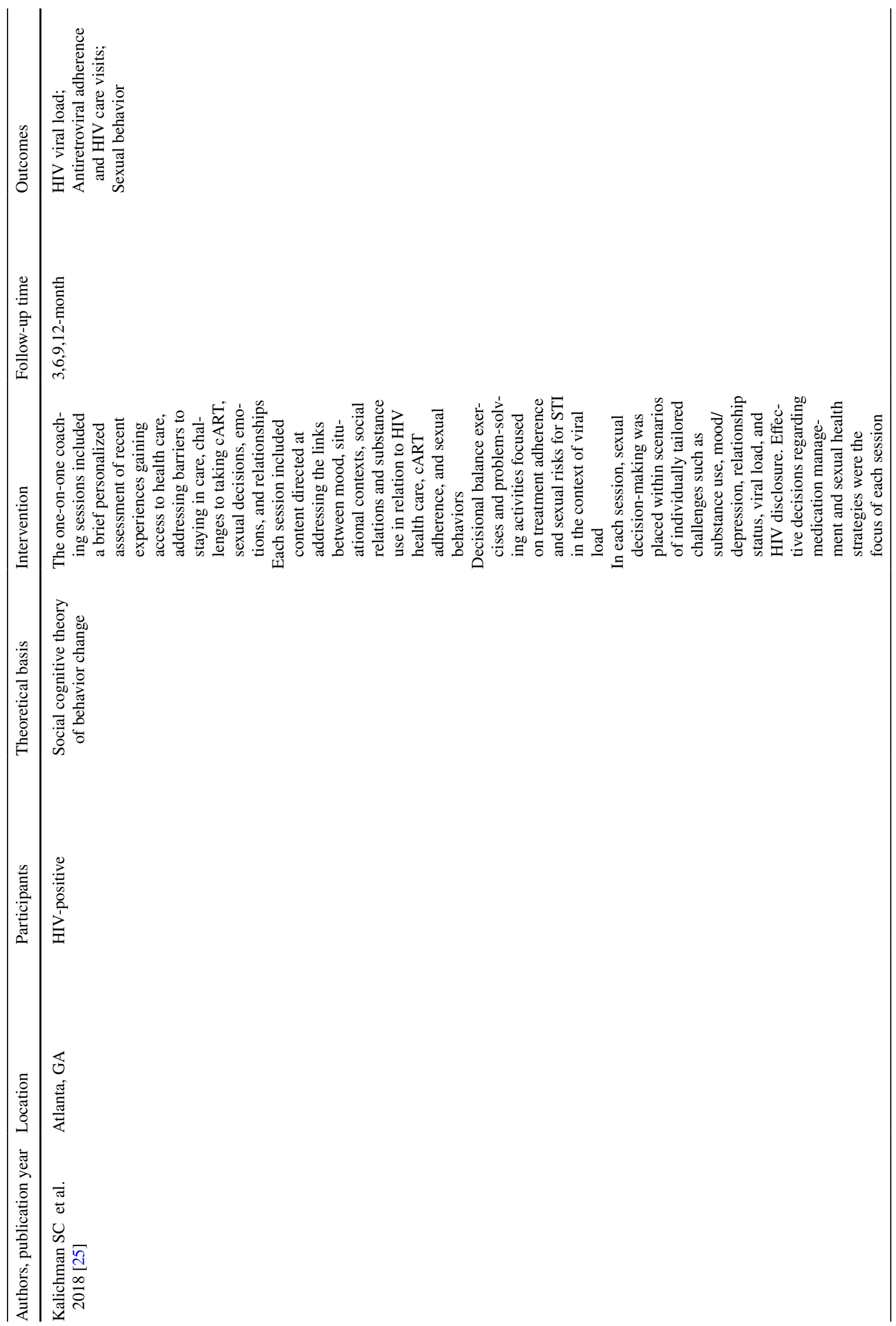




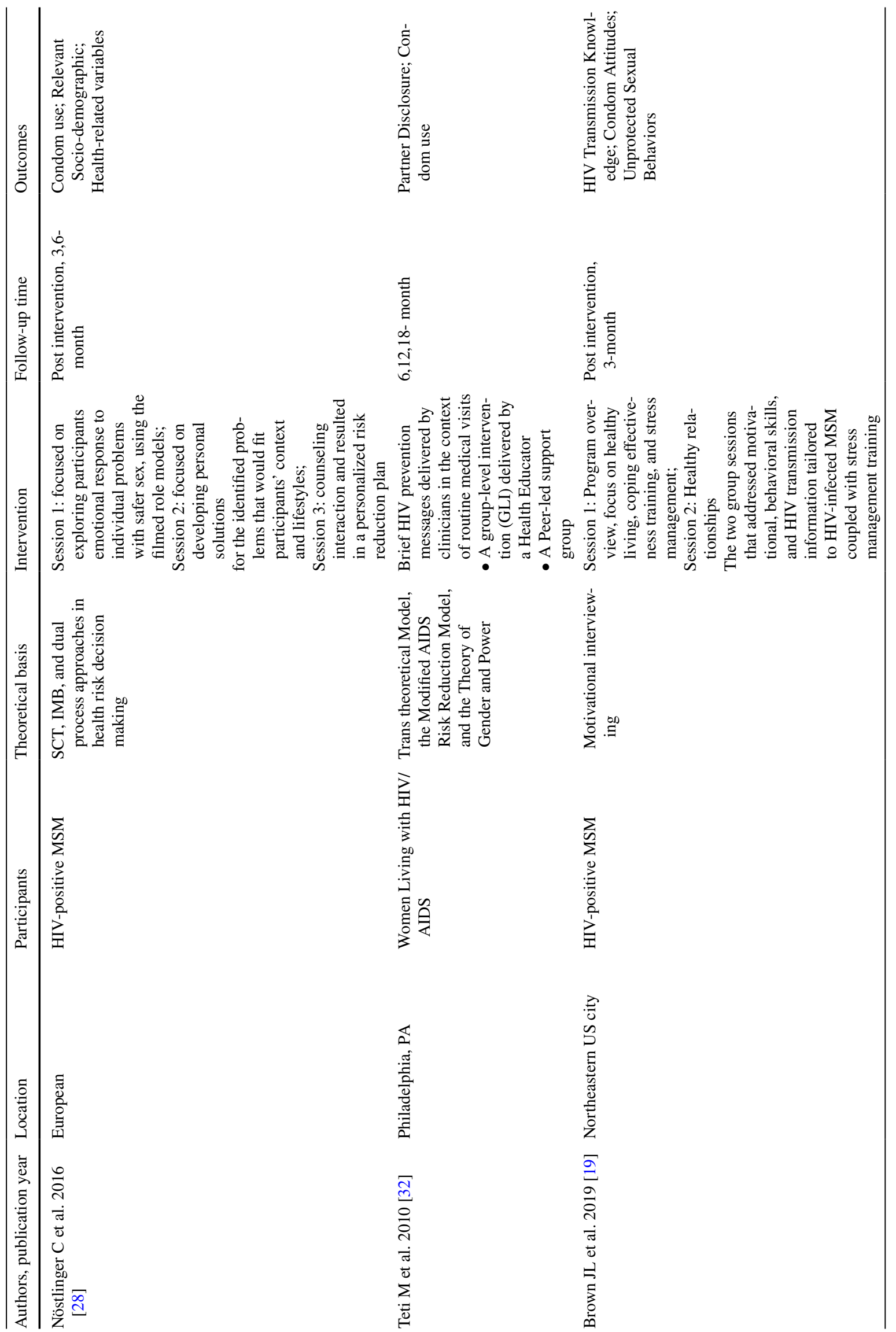




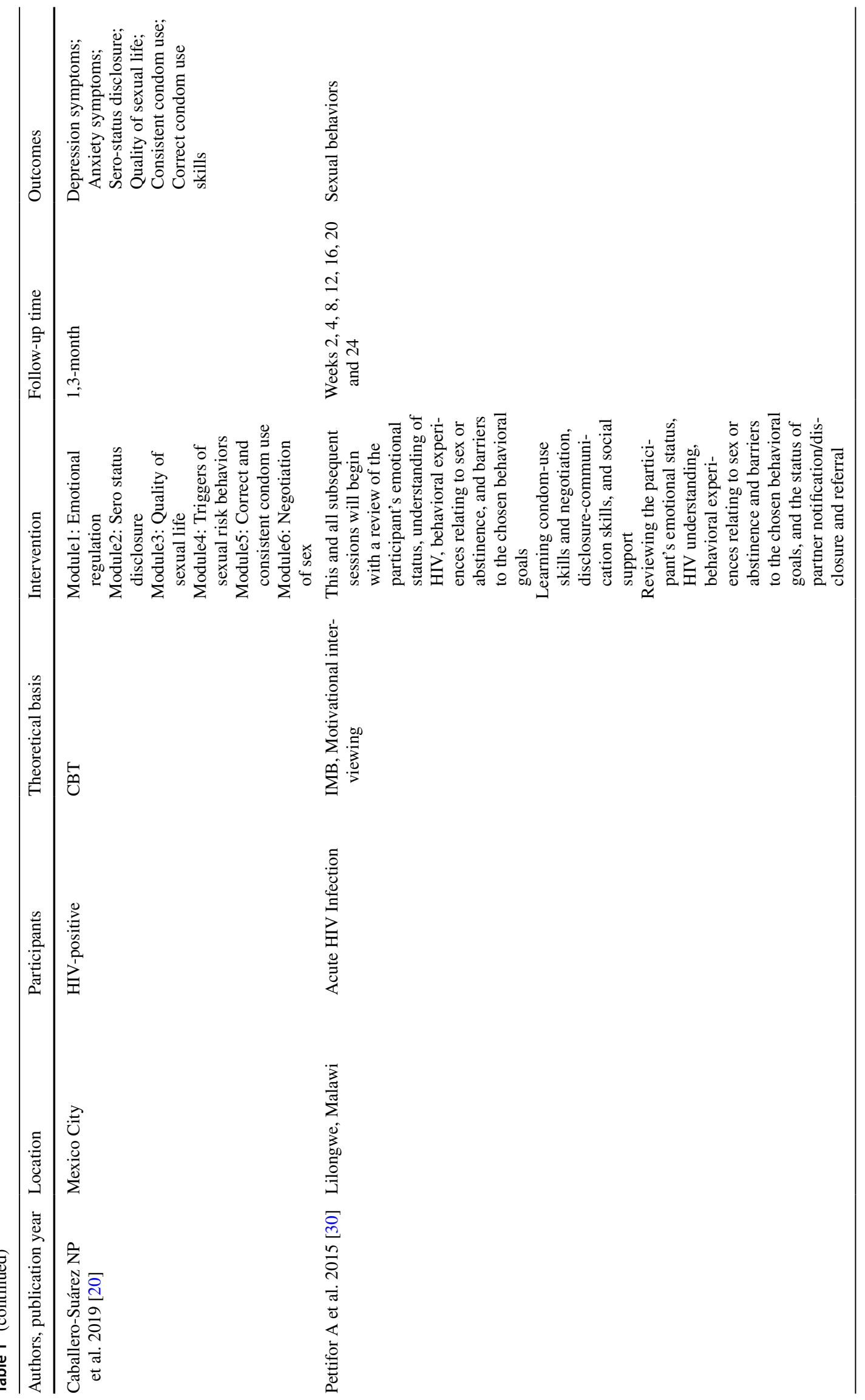


The studies differed substantially in their design. Eleven studies of them were randomized controlled trials (RCTs) with randomization at either the individual or group (facility/community) level [18, 21-28, 32, 33], while six studies employed non-randomized trials (pilot study/quasi-experimental study) or a randomized trial with no control [19, 20, 29-31, 34]. Studies varied in the number of interventions adopted, with fourteen reported a two-armed design, one reported a three-armed design, and two reported no control design.

Almost all studies had one or more underlying theoretical basis. Information, motivation and behavior skills model (IMB) [21, 26, 28, 30, 34], protection motivation theory/ social Cognitive theory $[22,25,27,28,31,33]$ were the first two theories that founded the basis of intervention, others including Motivational interviewing [19, 24, 30, 34], Transtheoretical Model [32], self-directed behavioral skills practice [34], relationship-oriented ecological framework [22, 27], Integrated Behavioral Model (IBM) [23], cognitivebehavior therapy (CBT) [20], Modified AIDS Risk Reduction Model [32], and the Theory of Gender and Power [32].

\section{Summary of Intervention Characteristics}

Interventions of seventeen studies showed in Table 1. Most interventions adopted face to face, but some interventions used multiple adaptations such as mobile phones or internetbased methods [23]. The online-intervention built forums for discussion on relevant topics, such as HIV prevention and treatment, dealing with stigma and relationships, safer sex behaviors, or provided space to upload and share personal videos, etc. Some interventions combined multiple intervention components, many of which based on IMB and MI model.

Almost all of these interventions involve changing behavior by changing thoughts, mainly focused on motivation activation and skill training, such as communicated risk awareness, thinking changes, status exposure, and condom use skills training. Some interventions also included counseling and strengthened/promoted the relationship between partners to improve condom use.

\section{Study Quality}

Table 2 presented information on quality assessment. More than half of all the studies were classified as low (9/17) quality regarding the overall quality classification. One study was classified as high quality. None of the studies met all quality criteria.

\section{Intervention Effects on Condom Use or Unprotected Sex Outcomes}

Table 3 showed detailed intervention effects on sexual behaviors. Included studies used different measures. Six studies provided proportions of condom use $[18,22,24,27$,

Table 2 Quality assessment of included studies

\begin{tabular}{|c|c|c|c|c|c|c|c|c|}
\hline Author & $\begin{array}{l}\text { Sequence } \\
\text { genera- } \\
\text { tion }\end{array}$ & $\begin{array}{l}\text { Allocation } \\
\text { conceal- } \\
\text { ment }\end{array}$ & $\begin{array}{l}\text { Blinding of } \\
\text { participants }\end{array}$ & $\begin{array}{l}\text { Incomplete } \\
\text { outcome } \\
\text { data }\end{array}$ & $\begin{array}{l}\text { Selective } \\
\text { outcome } \\
\text { reporting }\end{array}$ & $\begin{array}{l}\text { Availability } \\
\text { of treatment } \\
\text { manual }\end{array}$ & $\begin{array}{l}\text { Use of training for } \\
\text { therapy providers }\end{array}$ & $\begin{array}{l}\text { Quality } \\
\text { classifi- } \\
\text { cation }\end{array}$ \\
\hline Charles H. Klein & Low & Low & Low & Low & Unclear & Low & Low & Low \\
\hline Dean G. Cruess & Low & Unclear & Low & Low & Unclear & Unclear & Low & Low \\
\hline Jeffrey T. Parsons & NA & NA & High & Unclear & Unclear & Low & Low & Unclear \\
\hline Krishna C. Poudel & Unclear & Low & Unclear & Unclear & Unclear & Unclear & Low & Unclear \\
\hline Deborah L Jones & Unclear & Unclear & Unclear & Unclear & Unclear & Unclear & Unclear & Unclear \\
\hline Mark Williams & Low & Low & Unclear & Unclear & Unclear & Unclear & Unclear & Unclear \\
\hline Travis I. Lovejoy & Unclear & Low & Unclear & Unclear & Unclear & Low & Low & Low \\
\hline Trevor A. Hart & NA & NA & High & Unclear & Unclear & Low & Low & Unclear \\
\hline Nabila El-Bassel & Low & Low & Low & Low & Unclear & Low & Low & Low \\
\hline $\begin{array}{l}\text { Lisa B Hightow-Wei- } \\
\text { dman }\end{array}$ & Unclear & Unclear & Unclear & Unclear & Unclear & Low & Low & Unclear \\
\hline Sibongile Mashaphu & Low & Low & Low & Low & Unclear & Low & Low & Low \\
\hline Seth C. Kalichman & Low & Low & Low & Low & Unclear & Unclear & Low & Low \\
\hline Christiana Nöstlinger & Low & Low & High & High & Unclear & Unclear & High & High \\
\hline Michelle Teti & Low & Low & Unclear & Unclear & Unclear & Low & Low & Low \\
\hline Jennifer L. Brown & Unclear & Unclear & Unclear & Low & Unclear & Low & Low & Unclear \\
\hline $\begin{array}{l}\text { Nancy Patricia Cabal- } \\
\text { lero-Suárez }\end{array}$ & NA & NA & High & Unclear & Unclear & Low & Low & Low \\
\hline Audrey Pettifor & Low & Low & High & Unclear & Unclear & Low & Low & Low \\
\hline
\end{tabular}


Table 3 Sexual behaviors findings from included studies

\begin{tabular}{|c|c|c|c|}
\hline Variables & Author & \multicolumn{2}{|l|}{ Findings } \\
\hline \multicolumn{4}{|c|}{ Proportions of condom use } \\
\hline & Charles H. Klein & \\
\hline & & \multirow{3}{*}{\multicolumn{2}{|c|}{$\begin{array}{l}\text { Sex with All partners: } \text { mean difference }=0.33, P=0.002 \\
\text { HIV negative partners: mean difference }=0.31, P=0.04 \\
\text { HIV positive partners: mean difference }=0.48, P=0.003\end{array}$}} \\
\hline & & & \\
\hline & & & \\
\hline & Deborah L Jones & \multirow{3}{*}{\multicolumn{2}{|c|}{$\begin{array}{l}\text { Means: } \\
\text { Control: baseline: } 0.72 \text {, post intervention: } 0.70,6 \text {-month: } 0.63 \\
\text { Intervention: baseline: } 0.60 \text {, post intervention: } 0.73,6 \text {-month: } 0.57\end{array}$}} \\
\hline & & & \\
\hline & & & \\
\hline & Nabila El-Bassel & Intervention: & Control: \\
\hline & & Baseline: $0.44(0.38)$ & Baseline: $0.44(0.40)$ \\
\hline & & Post-intervention: $0.82(0.28)$ & Post intervention: $0.55(0.43)$ \\
\hline & & 6-month: $0.75(0.36)$ & 6-month: $0.56(0.43)$ \\
\hline & & 12-month: $0.72(0.38)$ & 12-month: $0.56(0.43)$ \\
\hline & Sibongile Mashaphu & \multirow{4}{*}{\multicolumn{2}{|c|}{$\begin{array}{l}\text { 3-month: } \\
\text { Intervention: } 73.68 \% \text { Control: } 37.50 \% \\
\text { 6-month: } \\
\text { Intervention: } 77.78 \% \text { Control: } 71.42 \%\end{array}$}} \\
\hline & & & \\
\hline & & & \\
\hline & & & \\
\hline & Nancy Patricia Caballero-Suárez & \multirow{9}{*}{\multicolumn{2}{|c|}{ 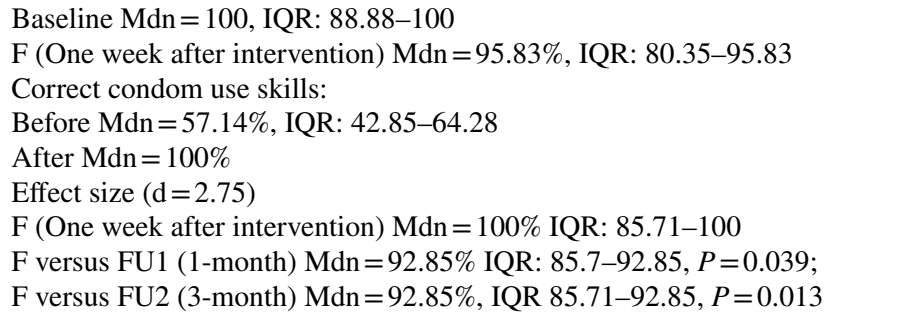 }} \\
\hline & & & \\
\hline & & & \\
\hline & & & \\
\hline & & & \\
\hline & & & \\
\hline & & & \\
\hline & & & \\
\hline & & & \\
\hline & Mark Williams & Intervention: & Control: \\
\hline & & Intake: $23 \%$ & Intake: $22 \%$ \\
\hline & & 3-month: $55 \%$ & 3-month: $39 \%$ \\
\hline & & 9-monh: $48 \%$ & 9-monh: $44 \%$ \\
\hline \multicolumn{4}{|c|}{ CAS* frequencies } \\
\hline & Jeffrey T. Parsons & \multirow{2}{*}{\multicolumn{2}{|c|}{$\begin{array}{l}\text { 3-month: } \\
\text { Means } \pm \text { d: } 9.50 \pm 1.66\end{array}$}} \\
\hline & & & \\
\hline & Trevor A. Hart & \multirow{10}{*}{\multicolumn{2}{|c|}{$\begin{array}{l}\text { Sex with Casual HIV-positive partners: } \\
\text { Baseline: } 61.5 \% \text {, post-intervention: } 46.2 \% \text { 3-month: } 42.3 \% \\
\text { Sex with Casual HIV-negative partners: } \\
\text { Baseline: } 17.3 \% \text {, post-intervention: } 21.2 \% \text {, 3-month: } 17.3 \% \text {; } \\
\text { Casual partners of unknown HIV-status: } \\
\text { Baseline: } 42.3 \% \text {, post-intervention: } 26.9 \% 3 \text {-month: } 21.1 \% \\
\text { Sex with Regular HIV-positive partner: } \\
\text { Baseline: } 32.8 \% \text {, post-intervention: } 28.9 \% \text { 3-month: } 25.0 \% \\
\text { Sex with Regular HIV-negative partner: } \\
\text { Baseline: } 1.9 \% \text {, post-intervention: } 1.9 \% \text { 3-month: } 0.0 \%\end{array}$}} \\
\hline & & & \\
\hline & & & \\
\hline & & & \\
\hline & & & \\
\hline & & & \\
\hline & & & \\
\hline & & & \\
\hline & & & \\
\hline & & & \\
\hline \multicolumn{4}{|c|}{ Proportion of CAS* } \\
\hline & Seth C. Kalichman & Intervention $(\mathrm{N}=250) \mathrm{M}(\mathrm{Se})$ & Comparison Condition $(\mathrm{N}=250)$ \\
\hline & & 2-month: $0.69(0.05)$ & $0.54(0.04)$ \\
\hline & & 4-month: $0.43(0.04)$ & $0.62(0.05)$ \\
\hline & & 5-month: $0.45(0.04)$ & $0.52(0.04)$ \\
\hline & & 6-month: $0.34(0.03)$ & $0.39(0.04)$ \\
\hline & & 7-month: $0.44(0.04)$ & $0.46(0.04)$ \\
\hline & & 9-month: $0.50(0.04)$ & $0.37(0.03)$ \\
\hline & & 10-month: $0.46(0.04)$ & $0.41(0.04)$ \\
\hline & & 12-month: $0.50(0.04)$ & $0.36(0.04)$ \\
\hline & Jennifer L. Brown & \multirow{2}{*}{\multicolumn{2}{|c|}{$\begin{array}{l}3 \text { months: } \\
\text { Intervention: } \mathrm{M} \text { (se) } 0.18(.05) \text { Control: } \mathrm{M}(\mathrm{se}) 0.27 \text { (.05), } \\
\text { Effect size }=0.02\end{array}$}} \\
\hline & & & \\
\hline
\end{tabular}


Table 3 (continued)

\begin{tabular}{|c|c|c|c|}
\hline \multirow[t]{2}{*}{ Variables } & \multirow{2}{*}{ Author } & \multicolumn{2}{|l|}{ Findings } \\
\hline & & $\begin{array}{l}\text { Intervention: } \\
\text { Baseline: } 36 \% \\
\text { Week 12: } 0 \% \\
\text { Week 24: } 0 \%\end{array}$ & $\begin{array}{l}\text { Control: } \\
54 \% \\
8 \% \\
0 \%\end{array}$ \\
\hline \multicolumn{4}{|l|}{ RR or OR } \\
\hline \multirow[t]{2}{*}{ Condom use RR } & Dean G. Cruess & \multicolumn{2}{|c|}{$\begin{array}{l}\text { 6-month: } \\
\text { Intervention vs. Control } \\
\text { Sex with All partners: incident rate ratios }(\text { IRR })=0.91(0.63-1.30) \\
\text { Sex with HIV-negative/unknown: }(\text { IRR })=0.33(0.20-0.53)\end{array}$} \\
\hline & Travis I. Lovejoy & \multicolumn{2}{|c|}{$\begin{array}{l}\text { 3-month follow-up, } \\
\text { 4-session MI participants vs. Controls: } \mathrm{OR}=3.15[1.02-9.72] \text {; } \\
\text { 4- session vs. 1-session MI conditions: } \mathrm{OR}=1.17[0.48-2.86] \\
\text { 1-session MI vs. Control conditions: } \mathrm{OR}=2.69[0.88-8.27] \\
\text { 6-month: } \\
\text { 4-session MI participants vs. Controls: } \mathrm{OR}=2.23[0.78-6.40] \\
\text { 4- session vs. 1-session MI conditions: } \mathrm{OR}=1.19[0.48-2.99] \\
\text { 1-session MI vs. Control conditions: } \mathrm{OR}=1.87[0.66-5.30]\end{array}$} \\
\hline CAS* RR & Lisa B Hightow-Weidman & $\begin{array}{l}\text { 3-months post-intervention: } \\
\text { HIV-positive partners: } \\
\text { Incidence ratio } 0.18,95 \% \text { CI: } 0.04,0.32 \\
\text { Either HIV-positive with undetectable } \\
\text { viral loads or HIV-negative/unknown: } \\
\text { Incidence ratio } 0.88,95 \% \text { CI: } 0.51,1.26\end{array}$ & $\begin{array}{l}\text { HIV-positive partners: } \\
\text { Incidence ratio 0.50, } 95 \% \text { CI: } 0.12 \text {, } \\
0.89 \\
\text { Either HIV-positive with undetect- } \\
\text { able viral loads or HIV-negative/ } \\
\text { unknown: } \\
\text { Incidence ratio } 0.87,95 \% \text { CI: } 0.51 \text {, } \\
1.23\end{array}$ \\
\hline Condom use OR & Christiana Nöstlinger & \multicolumn{2}{|c|}{$\begin{array}{l}3 \text { month vs. baseline: } \\
\text { Intervention: OR =6.77, 95\%CI: } 2.48-18.52 \\
\text { Control: OR =1.77, 95\%CI: } 0.72-4.32 P=0.03 \\
\text { 6-month vs. baseline: } \\
\text { Intervention: } \mathrm{OR}=5.46,95 \% \text { CI: } 2.16-13.79 \\
\text { Control: } \mathrm{OR}=2.53,95 \% \text { CI: } 0.98-6.52 P=0.19\end{array}$} \\
\hline $\begin{array}{l}\text { Proportion of condom use } \\
\text { difference OR }\end{array}$ & Michelle Teti & \multicolumn{2}{|c|}{$\begin{array}{l}\text { Baseline difference: OR: } 4.58(0.85,24.74) \\
\text { 6-Month difference: OR: } 17.13(2.96,99.10) \\
\text { 12-Month difference: OR: } 1.52(0.25,9.24) \\
\text { 18-Month difference: OR: } 270.04(24.53,2971.94)\end{array}$} \\
\hline \multicolumn{4}{|c|}{ Condom use intention net difference } \\
\hline & Krishna C. Poudel & \multicolumn{2}{|l|}{$\begin{array}{l}\text { Post intervention: } \\
\text { Regular partners: } 0.60, P<0.001 ; \\
\text { HIV positive: } 0.36, P=0.005 ; \\
\text { HIV negative: } 0.11, P=0.34 ; \\
\text { HIV status unknown: } 0.05, P=0.65\end{array}$} \\
\hline
\end{tabular}

CAS Condom less Anal sex

$I R R$ Incidence risk ratio

30, 32], five studies used RR or OR as the intervention effect size [21, 23, 26, 28, 32], and others provided proportions of condomless anal sex (CAS) [19, 25, 30], frequencies of CAS [29, 34], and condom use intention [31]. These studies' follow-up time points were generally post-intervention, three and six months after completing the intervention. And one study used 'Treatment as Prevention' intervention and follow up almost every month after the intervention [25].

We did meta-analysis with three interventions, which had the same measure for proportions of condom use, with means (standard variations) provided in both arms [18,
22, 24]. Heterogeneity was present in these three studies $\left(I^{2}=56 \%\right)$, so the random effect model was used (Fig. 2). The pooled results showed that the condom use proportion increased in the experimental group after intervention compared with the control group (Random SMD (Standard Mean Difference): 0.36; 95\% CI: 0.06, 0.65). The intervention mainly contained emotional regulation, increasing communication with sexual partners, and teaching condom use skills.

There were also trials with more than one intervention arm, Travis I. Lovejoy's study used 4-session intervention and 


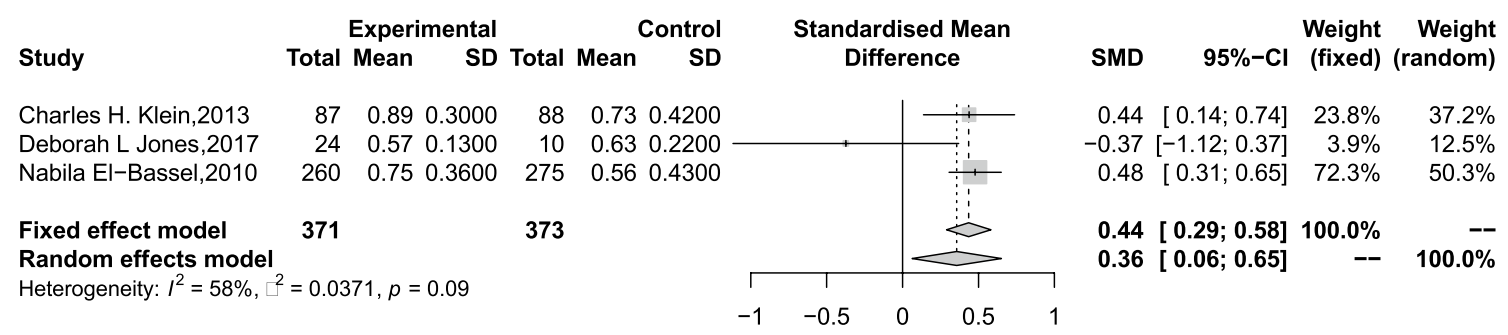

Fig. 2 Forest plot of effect size: impact of prevention interventions on proportions of condom uses

1-session MI to intervene with the participants. They found significant effects in 4-session group versus the control group for condom use in 3-month $(O R=3.15,95 \% C I$ : 1.02-9.72) but not significant in 6-month follow-up $(O R=2.23,95 \% \mathrm{CI}$ : 0.78-6.40). In addition, no differences were observed between participants in the 1-session and control conditions or the 4-session and 1-session MI conditions at 3-month $(O R=2.69$, 95\% CI: 0.88-8.27 and $O R=1.17,95 \%$ CI: 0.48-2.86, respectively) or 6-month follow-up ( $O R=1.87,95 \%$ CI: 0.66-5.30 and $O R=1.19,95 \% C I: 0.48-2.99$, respectively). It was noteworthy that the intervention effect decreased over time after the intervention [26].

Some studies compared condom use between different sex partners, including HIV-positive, HIV-negative, or unknown status. It showed that there were differences when had sex with HIV-positive partners compared with HIV-negative or unknown status partners [18, 21, 23, 34]. In Trevor A. Hart et al. study, they aimed to use IMB, MI to enhance condom use among HIV-positive MSM. The results showed that the condomless anal sex decreased at post-intervention and 3-month follow-up with any type of sexual partners. Besides, during the sex with casual partners who were HIV-negative or unknown, the condomless anal sex decreased largely [34]. Both HIVpositive couples might not use condoms during sex, so interventions targeting multiple HIV infections or drug resistance might be an effective intervention in helping increase condom use for both HIV-positive couples. Results also showed that condomless sex frequencies reduced for HIV-negative or unknown infection status partners, which were the significant factor for HIV transmission decreasing [35, 36]. Mark Williams's study included the risks and consequences of HIV/ STD infection/reinfection, HIV transmission to sex partners, and risks when both sex partners were HIV-positive [33]. In summary, our finding provided additional evidence for psychological intervention was helpful for HIV risk behaviors reduction, which was significant for public health implications and HIV reduction.

\section{Discussion}

This systematic review and meta-analysis provided support for conducting psychological interventions on risky sexual behaviors among people living with HIV/AIDS. For the theory basis, most studies used the IMB model and MI. The IMB model combined with HIV prevention information, motivation, and behavioral skills to change sexual risk behaviors. The IMB model was an empirically supported model for sexual risk reduction and mental health problem reduction. The intervention content involved in this paper was almost combined with HIV-related knowledge, mental health management, behavioral skills, and communication or how to disclose with sexual partners.

Overall, these interventions had positive effects on reducing sexual risk, for example, psychological interventions significantly improved the proportion of condom use. However, in most studies, effects on condom use increased post-intervention, then the effect declined with time. Effect of psychological or behavioral intervention was peak immediately after the completion of the intervention, then decreased over time, which indicated that the intervention needs to be permanent and normalized to benefit PLWH consistently.

One study combined psychological interventions into a mobile phone and internet-based platform. This study also showed a positive effect on condom use: for HIV-positive partners, the RR for CAS was 0.50 (95\% CI: 0.12, 0.89) compared with the control group, and the RR for CAS was 0.87 (95\% CI: $0.51,1.23)$ for either HIV-positive with undetectable viral loads or HIV-negative/unknown partners [23]. Mobile health intervention studies had shown feasibility and efficacy to improve mental health in different populations in recent years [37-41]. There were trends that mobile health (mHealth) intervention on improving 
mental health because of its convenience and accessibility. Moreover, it may be imperative to develop effective interventions to improve mental health and quality of life for PLWH considering the current COVID-19 epidemic.

Considering the decrease of the intervention effects as time passed by, it was important to investigate whether these interventions could be administered easily, such as conducted at a community level, as one part of normal care for PLWH, or intervened by mHealth. One study used a mutual recovery method based on CBT among community-dwelling adults with depressive symptoms in China in 2012, and the decrease in depressive symptoms was observed immediately after the intervention [42]. This study reminded us to explore the non-personal intervention methods to change mental health or behaviors.

\section{Limitations}

The sexually related behaviors were not merely condom use; also included commercial sex, more than one sex partner, etc. This review set condom use as the outcome of the psychological interventions, which may not be able to conclude the comprehensive effects of the intervention. Secondly, data used in this review was only derived from literature published in English, with non-English databases excluded consciously. Thirdly, although this review showed the efficacy of psychological health interventions on behavior change, we did not further compare the effectiveness of the different psychosocial interventions to find the most effective one. Thus, more research was needed to better understand these findings.

\section{Conclusion}

This systematic review and meta-analysis found that psychological interventions might positively affect the condom use of PLWH. Based on this meta-analysis results, it is essential to incorporate psychosocial therapies into the routine care of PLWH to avoid the transient effects of the concerned intervention.

\section{Appendix}

[Search terms included (hiv [mesh] or hiv infection [mesh] or hiv [tiab] or aids [tiab] or acquired human immunodeficiency syndrome[tiab] or human immunodeficiency virus[tiab]) AND (psychotherapy [mesh] or mental health services [mesh] or self-care [mesh] or self-help groups [mesh] or telemedicine [mesh] or therapy, computer assisted [mesh] or psychotherap* [tiab] or psychological therap* [tiab] or psychological treatment* [tiab] or psychological intervention* [tiab] or counsel* [tiab] or cbt [tiab] or behavior therap* [tiab] or behaviour therap*[tiab] or interpersonal therap* [tiab] or coping [tiab] or peer support [tiab] or social support [tiab] or problem solving [tiab] or stress manage* [tiab] or self-help [tiab] or internet therap* [tiab] or online therap* [tiab] or psychoed* [tiab] or training [tiab] or exposure therap* [tiab]relaxation [tiab] or mindfulness [tiab] or reinforcement [tiab] or commitment therap* [tiab] or case manage* [tiab] OR depression [mesh] OR depress* [tiab] OR anxiety [mesh] OR anxi* [tiab] OR fear [tiab] OR quality of life [mesh] OR quality-of-life [tiab] OR well-being [tiab] OR stress, psychological [mesh] OR stress* [tiab] OR distress [tiab] OR mental health [tiab]) AND (condom use[tiab] or safe sex[tiab] or condom*[tiab]) AND ((clinicaltrial[Filter] OR randomizedcontrolledtrial[Filter]) AND (y_10[Filter]) AND (humans[Filter]) AND (english[Filter])).]

\section{Compliance with Ethical Standards}

Conflict of interest The authors declare that they have no conflict of interest.

Research Involving Human Participants and/or Animals This article does not contain any studies with human participants or animals performed by any of the authors.

Informed Consent Informed consent did not apply to our study, as it contained no studies with human participants performed by any of the authors.

\section{References}

1. Yin L, Wang N, Vermund SH, Shepherd BE, Ruan Y, et al. Sexual risk reduction for HIV-infected persons: a meta-analytic review of " "positive prevention"” randomized clinical trials. PLoS ONE. 2014;9(9):e107652. https://doi.org/10.1371/journal.pone.01076 52.

2. Niu L, Luo D, Liu Y, Silenzio VMB, Xiao S. The mental health of people living with HIV in China, 1998-2014: a systematic review. PLoS ONE. 2016;11:e153489.

3. Walkup J, Blank MB, Gonzalez JS, Schwartz R, Brown L, et al. The impact of mental health and substance abuse factors on HIV prevention and treatment. J Acquir Immune Defic Syndr. 2008;47(Suppl 1):S15.

4. Shen H, Zou H, Huang S, Liu F, Zhao P, Chen L, Zhang Ye, Luo $\mathrm{X}$, Tang W, Zheng H, Yang B. Depression and HIV risk behaviors among female sex workers in Guangdong, China: a multicenter cross-sectional study. Biomed Res Int. 2016. https://doi. org/10.1155/2016/6986173.

5. Hong Y, Li X, Fang X, Zhao R. Depressive symptoms and condom use with clients among female sex workers in China. Sexual Health. 2007;4(2):99-104.

6. Berger BE, Ferrans CE, Lashley FR. Measuring stigma in people with HIV: psychometric assessment of the HIV stigma scale. Res Nurs Health. 2001;24:518-29. 
7. Jonathon Rendina H, Gamarel KE, Pachankis JE, Ventuneac A, Grov C, Parsons JT. Extending the minority stress model to incorporate HIV-positive gay and bisexual men's experiences: a longitudinal examination of mental health and sexual risk behavior. Ann Behav Med. 2017;51(2):147-58. https://doi. org/10.1007/s12160-016-9822-8.

8. van Luenen S, Garnefski N, Spinhoven P, Spaan P, Dusseldorp E, Kraaij V. The benefits of psychosocial interventions for mental health in people living with HIV: a systematic review and meta-analysis. AIDS Behav. 2018;22(1):9-42.

9. Spies G, Asmal L, Seedat S. Cognitive-behavioural interventions for mood and anxiety disorders in HIV: a systematic review. J Affect Disord. 2013;150(2):171-80.

10. Johnson BT, Carey MP, Chaudoir SR, Reid AE. Sexual risk reduction for persons living with HIV: research synthesis of randomized controlled trials, 1993 to 2004. J Acquir Immune Defic Syndr. 2006;41:642-50.

11. Weller SC, Davis-Beaty K. Condom effectiveness in reducing heterosexual HIV transmission. Cochrane Database Syst Rev. 2002. https://doi.org/10.1002/14651858.CD003255.

12. Crosby RA, DiClemente RJ, Charnigo R, et al. A brief, clinicbased, safer sex intervention for African American men at-risk of HIV acquisition: a randomized controlled trial. Am J Public Health. 2009;99:S96-103.

13. Crosby RA. State of condom use in HIV prevention science and practice. Curr HIV/AIDS Rep. 2013;10:59-64. https://doi. org/10.1007/s 11904-012-0143-7.

14. Crosby RA, Cates W. Condom use: still a sexual health staple. Sex Health. 2012;9:1-3. https://doi.org/10.1071/SH11111.

15. Moher D, Liberati A, Tetzlaff J, Altman DG. Preferred reporting items for systematic reviews and meta-analyses: the PRISMA statement. PLoS Med. 2009;6:e1000097.

16. Higgins JPT, Altman DG, Gotzsche PC, Juni P, Moher D, Oxman AD, et al. The cochrane collaboration's tool for assessing risk of bias in randomised trials. BMJ. 2011;343:9.

17. Crepaz N, Baack BN, Higa DH, Mullins MM. Effects of integrated interventions on transmission risk and care continuum outcomes in persons living with HIV: meta-analysis, 19962014. AIDS. 2015;29(18):2371-83. https://doi.org/10.1097/ QAD.0000000000000879.

18. Klein CH, Lomonaco CG, Pavlescak R, Card JJ. WiLLOW: reaching HIV-positive African-American women through a computer-delivered intervention. AIDS Behav. 2013;17(9):3013-23.

19. Brown JL, Vanable PA, Bostwick RA, Carey MP. A pilot intervention trial to promote sexual health and stress management among HIV-infected men who have sex with men. AIDS Behav. 2019;23(1):48-59.

20. Caballero-Suarez NP, Iglesias MC, Estrada ER, Teran GR, Rosas AR. Effects of cognitive-behavioural therapy on anxiety, depression and condom use in people with HIV in Mexico city: a pilot study. Psychol Health Med. 2019;24(1):115-25.

21. Cruess DG, Burnham KE, Finitsis DJ, Goshe BM, Strainge L, Kalichman M, et al. A randomized clinical trial of a brief internet-based group intervention to reduce sexual transmission risk behavior among HIV-positive gay and bisexual men. Ann Behav Med. 2018;52(2):116-29.

22. El-Bassel N, Jemmott JB, Landis JR, Pequegnat W, Wingood GM, Wyatt GE, et al. National institute of mental health multisite Eban HIV/STD prevention intervention for African American HIV Serodiscordant couples a cluster randomized trial. Arch Intern Med. 2010;170(17):1594-601.

23. Hightow-Weidman LB, LeGrand S, Muessig KE, Simmons RA, Soni K, Choi SK, et al. A randomized trial of an online risk reduction intervention for young black MSM. AIDS Behav. 2019;23(5):1166-77.
24. Jones DL, Echenique M, Potter J, Rodriguez VJ, Weiss SM, Fischl MA. Adolescent girls and young women living with HIV: preconception counseling strategies. Int J Womens Health. 2017;9:657-63.

25. Kalichman SC, Cherry C, Kalichman MO, Eaton LA, Kohler JJ, Montero C, et al. Mobile health intervention to reduce HIV transmission: a randomized trial of behaviorally enhanced HIV treatment as prevention (B-TasP). JAIDS. 2018;78(1):34-42.

26. Lovejoy TI, Heckman TG. Depression moderates treatment efficacy of an HIV secondary-prevention intervention for HIV-positive late middle-age and older adults. Behav Med. 2014;40(3):124-33.

27. Mashaphu S, Wyatt GE, Zhang M, Mthiyane T, Liu H, Gomo E. Effectiveness of an HIV-risk reduction intervention to reduce HIV transmission among serodiscordant couples in Durban, South Africa. A randomized controlled trial. AIDS Care. 2020;32(5):537-45.

28. Nostlinger C, Platteau T, Bogner J, Buyze J, Dec-Pietrowska J, Dias S, et al. Implementation and operational research: computerassisted intervention for safer sex in HIV-positive men having sex with men: findings of a European randomized multi-center trial. JAIDS. 2016;71(3):E63-72.

29. Parsons JT, Rendina HJ, Moody RL, Gurung S, Starks TJ, Pachankis JE. Feasibility of an emotion regulation intervention to improve mental health and reduce HIV transmission risk behaviors for HIV-positive gay and bisexual men with sexual compulsivity. AIDS Behav. 2017;21(6):1540-9.

30. Pettifor A, Corneli A, Kamanga G, McKenna K, Rosenberg NE, Yu X, et al. HPTN 062: a pilot randomized controlled trial exploring the effect of a motivational-interviewing intervention on sexual behavior among individuals with acute HIV infection in Lilongwe, Malawi. PLoS ONE. 2015;10(5):e124452.

31. Poudel KC, Buchanan DR, Poudel-Tandukar K. Effects of a community-based HIV risk reduction intervention among HIV-positive individuals: results of a quasi-experimental study in Nepal. AIDS Educ Prev. 2015;27(3):240-56.

32. Teti M, Bowleg L, Cole R, Lloyd L, Rubinstein S, Spencer S, et al. A mixed methods evaluation of the effect of the protect and respect intervention on the condom use and disclosure practices of women living with HIV/AIDS. AIDS Behav. 2010;14(3):567-79.

33. Williams M, Bowen A, Atkinson JS, Nilsson-Schnnesson L, Diamond PM, Ross MW, et al. An assessment of brief group interventions to increase condom use by heterosexual crack smokers living with HIV infection. AIDS. 2012;24(2):220-31.

34. Hart TA, Stratton N, Coleman TA, Wilson HA, Simpson SH, Julien RE, et al. A pilot trial of a sexual health counseling intervention for HIV-positive gay and bisexual men who report anal sex without condoms. PLoS ONE. 2016;11(4):e0152762.

35. Crepaz N, Marks G, Liau A, et al. HIV/AIDS prevention research synthesis (PRS) team prevalence of unprotected anal intercourse among HIV-diagnosed MSM in the United States: a meta-analysis. AIDS. 2009;23(13):1617-29.

36. Pantalone DW, Huh D, Nelson KM, Pearson CR, Simoni JM. Prospective predictors of unprotected anal intercourse among HIVseropositive men who have sex with men initiating antiretroviral therapy. AIDS Behav. 2014;18(1):78-87.

37. Wahle F, Kowatsch T, Fleisch E, Rufer M, Weidt S. Mobile sensing and support for people with depression: a pilot trial in the wild. JMIR Mhealth Uhealth. 2016;4:e111.

38. East ML, Havard BC. Mental health mobile apps: from infusion to diffusion in the mental health social system. JMIR Ment Health. 2015;2:e10.

39. Berrouiguet S, Baca-Garcia E, Brandt S, Walter M, Courtet P. Fundamentals for future mobile-health (mhealth): a systematic review of mobile phone and web-based text messaging in mental health. J Med Internet Res. 2016;18:e135. 
40. Berry N, Lobban F, Emsley R, Bucci S. Acceptability of interventions delivered online and through mobile phones for people who experience severe mental health problems: a systematic review. J Med Internet Res. 2016;18:e121.

41. Free C, Phillips G, Galli L, Watson L, Felix L, Edwards P, et al. The effectiveness of mobile-health technology-based health behaviour change or disease management interventions for health care consumers: a systematic review. PLOS Med. 2013;10:e1001362.
42. Wang C, Hua Y, Hua Fu, et al. Effects of a mutual recovery intervention on mental health in depressed elderly community-dwelling adults: a pilot study. BMC Public Health. 2017. https://doi. org/10.1186/s12889-016-3930-z.

Publisher's Note Springer Nature remains neutral with regard to jurisdictional claims in published maps and institutional affiliations. 